\section{Sensitivity in Multiobjective Programming \\ by Differential Equations Methods. \\ The case of Homogeneous Functions}

A. Balbás ${ }^{1}$ and P. Jiménez Guerra ${ }^{2}$

${ }^{1}$ Departamento de Economía de la Empresa. Universidad Carlos III de Madrid.

c) Madrid 126. 28903 Getafe (Madrid, Spain).

${ }^{2}$ Departamento de Matemáticas Fundamentales. Facultad de Ciencias. UNED.

c) Senda del Rey sln. 28040 Madrid (Spain).

Abstract. The purpose of this paper is to characterize for convex multiobjective programming, the situations in which the sensitivity with respect to the right side vector of the constraints can be obtained as a solution of a dual program.

Key words and phrases. Convex program, dual program, homogeneous function, sensitivity.

\section{Introduction}

It is well known that for convex scalar minimization programs and under very general conditions, the differential of the optimum value of the objective with respect to the right side vector of the constraints is a solution of a dual program. The extensions of this result to the multiobjective programming have been slight in extensions of this result to the multiobjective programming have been slight in
general, although we can refer for instance to [9] where the problem is studied for finite dimensional linear programs assuming that the decisor chooses always optimum solutions which are also basic feasible solutions.

Many papers have stated important contributions to the development of a duality theory for the multiobjective convex programming (see for instance [10], [13], [15] and [17]) but a result about sensitivity similar to that of the scalar case has never been obtained although several sets have been studied which can be determined by duality properties and which contain the mentioned differential (see for instance [14]). In [3] it is proved that in general for a multiobjective linear program its sensitivity depends not only on the dual solution but also on the differential of this dual solution. It is also proved that this result is a genuine extension of the standard one in scalar programming, and several examples are presented where the differential of the dual solution does not vanish. Last results have been extended for convex programs in [4] and for differential programs in [2], where the sensitivity is measured with respect to any parameter appearing in the program (and not only with respect to the right side vector of the constraints).

We use here the dual introduced in [4] since it extends the classic ones of the linear multiobjective programming and the scalar convex programming. In Section 3 the situations in which the primal sensitivity coincides with the dual solution are characterized. The main result is Theorem 3.4 which states a condition of homogeneity which solves completely the problem using the Euler's Formula (which characterizes the homogeneous functions). Afterwards it is studied the particular case of programs defined by homogeneous functions obtaining in this case that the dual solution measures the sensitivity if and only if the optimum value of the objective is an homogeneous function of the right side vector of the constraints (see Theorem 3.7). An important particular case is the linear programming which is studied in Section 4 where Theorem 4.2 characterizes the property when the optimum solution is chosen to be a convex linear combination of property when the optimum solution is chosen to be a convex linear combination of dual solution if and only if the weights applied over every basic feasible solution are homogeneous functions of degree zero.

\section{Preliminaries and notations}

Let $\Omega$ be a convex subset of a Banach space $\mathrm{X}, \mathrm{Y}$ a Banach space ordered by a closed convex pointed cone $Y_{+}, Z$ a Banach lattice with positive cone $Z_{+}$and $W$ an order complete Banach lattice with positive cone $W_{+}$. Let also consider two convex functions $\mathrm{f}: \Omega \rightarrow \mathrm{Y}$ and $\mathrm{g}: \Omega \rightarrow \mathrm{Z}$, and a surjective linear and continuous mapping $\mathrm{T}: \mathrm{Y} \rightarrow \mathrm{W}$ such that $\mathrm{T}\left(\mathrm{Y}_{+}-\{0\}\right) \subseteq \mathrm{W}_{+}-\{0\}$ and $\operatorname{Ker} \mathrm{T}$ has a topological supplement $\mathrm{YT}_{\mathrm{T}}$ (in $\mathrm{Y}$ ) (last condition holds in particular, if $\mathrm{Y}$ is a Hilbert space or $\mathrm{W}$ is finite dimensional). Let us denote by $\mathrm{T}^{*}$ the restriction of $\mathrm{T}$ to $Y_{T}$ (it follows from the open mapping theorem that $T^{*-1}$ is continuous), by $\pi$ the natural projection from $Y$ onto $\operatorname{KerT}$ and by $L(Z, W)$ the space of all linear and continuous functions from $\mathrm{Z}$ into $\mathrm{W}$, endowed with the usual norm and order. Similar notations will be used in other similar cases. Finally let $B$ be an open subset of $Z$ and consider for every $b \in B$ the following multiobjective minimization program:

$$
\begin{gathered}
\operatorname{Min} f(x) \\
x \in \Omega, g(x) \leq b
\end{gathered}
$$

For every $L \in L(Z, W)$, we write $L \in \Gamma_{T}$ if $L \geq 0$ and $\{\mathrm{Tf}(\mathrm{x})+\operatorname{Lg}(\mathrm{x})$ : $x \in \Omega\}$ is an order bounded from below subset of $W$, and in this case its infimum will be denote by $\varphi(\mathrm{T}, \mathrm{L})$. Following [4], for every $\mathrm{b} \in \mathrm{B}$ and $\mathrm{G} \in L(\mathrm{Z}, \mathrm{Y})$ such that $T G \in \Gamma T$ we consider the dual function $\psi(T, G)=T^{*}-1 \varphi(T, T G)-G(b)$ and the dual program

$$
\operatorname{Max} \psi(T, G)
$$

$\mathrm{G} \in L(\mathrm{Z}, \mathrm{Y}), \mathrm{TG} \in \Gamma_{\mathrm{T}}$

We say that $x_{b} \in \Omega$ is a T-optimal solution of $(2,1 b)$ if $x_{b}$ is a feasible solution of this program and $\operatorname{Tf}(x) \geq \operatorname{Tf}\left(x_{b}\right)$ for every feasible solution $x \in \Omega$. Analogously a feasible solution $G_{b} \in L(Z, Y)$ of $(2,2 b)$ is said to be a T-optimal solution of this program if $T \psi(T, G) \leq T \psi\left(T, G_{b}\right)$ for every feasible solution $\mathrm{G} \in L(Z, Y)$. If $\mathrm{x}_{\mathrm{b}}$ is a primal $T$-optimal solution, $\mathrm{G}_{\mathrm{b}}$ is a dual T-optimal solution and $f\left(x_{b}\right)=\psi\left(T, G_{b}\right)$ then $x_{b}$ and $G_{b}$ are said to be associated solutions. Several conditions for the existence of dual T-optimal solutions associated with a primal T-optimal solution can be fond in [4].

\section{Homogeneity conditions}

Throughout this paper we will suppose that for every $b \in B$ there exists a 
T-optimal solution $x_{b}$ of $(2,1 b)$ and $F: B \rightarrow Y$ will be the function defined by $F(b)=f\left(x_{b}\right)$ for every $b \in B$. Theorem 12 of [4] states that, "if there exists a Fréchet differentiable function $\mathbf{G}: \mathrm{B} \rightarrow L(\mathrm{Z}, \mathrm{Y})$ such that $\mathbf{G}(\mathrm{b})$ is a T-optimal solution of $(2,2 b)$ associated to $x_{b}$ for every $b \in B$, then $F$ is also Fréchet differentiable (on $B$ ) and $F^{\prime}(b, z)=-G_{b}(z)-\pi\left(G^{\prime}(b, z)(b)\right)$ for every $b \in B$ and $z \in Z$ ", where $F^{\prime}(b, z)$ and $G^{\prime}(b, z)$ denote the images of $z$ by the Fréchet differentials at $b$ of $F$ and $\mathbf{G}$, respectively, and $\mathbf{G}^{\prime}(b, z)(b)$ is the image of b by $\mathbf{G}^{\prime}(b, z)$. Throughout the paper $F^{\prime}(b,-)$ will denote the Fréchet differential of $F$ at $b \in B$.

In this Section we characterize the situations where the differential $F^{\prime}(b,-)$ is obtained as a dual T-optimal solution associated to $x_{b}$, for every $b \in B$.

Lemma 3.1. Let us assume that $F$ is Fréchet differentiable on $B$ and at least one of the following conditions holds:

3.1.1. B is convex and $\mathrm{g}(\Omega) \subseteq \mathrm{B}$.

3.1.2. There exists a continuous function $\mathbf{G}: \mathbf{B} \rightarrow L(\mathrm{Z}$. $\mathrm{Y})$ such that $\mathbf{G}(\mathrm{b})$ is a

T-optimal solution of $(2,2 b)$ associated to $x_{b}$, for every $b \in B$.

Then, for every $b \in B,-F^{\prime}(b,-)$ is a dual $T$-optimal solution and

$$
\varphi\left(T,-T F^{\prime}(b,-)\right)+T F^{\prime}(b, b)=T F(b) .
$$

Proof. Suppose that 3.1.1 holds and let $b \in B$ and $z \in Z_{+}$be. Then, there exists $\varepsilon>0$ such that $b+t z \in B$ for every $0 \leq t<\varepsilon$, and since $x_{b}$ is a feasible solution of

$(2,1(b+t z))$, we have that $T F(b+t z) \leq T F(b)$ and

$$
T F^{\prime}(b, z)=\lim _{t \rightarrow 0} \frac{T F(b+t z)-T F(b)}{t} \leq 0 .
$$

Let us see now that TF is a convex function. In fact, if $b, b^{\prime} \in B, \alpha, \alpha^{\prime} \geq 0$ and $\alpha+\alpha^{\prime}=1$, then since $\mathrm{g}$ is convex, we have that $g\left(\alpha x_{b}+\alpha^{\prime} x_{b^{\prime}}\right) \leq \alpha g\left(x_{b}\right)+\alpha^{\prime} g\left(x_{b^{\prime}}\right) \leq \alpha b+\alpha^{\prime} b^{\prime}$

and therefore $\operatorname{TF}\left(\alpha b+\alpha^{\prime} b^{\prime}\right) \leq \operatorname{Tf}\left(\alpha x_{b}+\alpha^{\prime} x_{b^{\prime}}\right) \leq \alpha \operatorname{TF}(b)+\alpha^{\prime} \operatorname{TF}\left(b^{\prime}\right)$.

Consider now $x \in \Omega$ and $h=g(x)-b$, then $b+h=g(x) \in B$ and for every $0 \leq \mathrm{t} \leq 1$ we have that

$\operatorname{TF}(b+t h)=\operatorname{TF}((1-t) b+t(h+b)) \leq(1-t) \operatorname{TF}(b)+t T F(h+b)$, from where we obtain that

$T F(b+t h)-T F(b)-T F^{\prime}(b, t h) \leq t\left[T F(b+h)-T F(b)-T F^{\prime}(b, h)\right]$. Therefore

$$
\frac{\mathrm{TF}(\mathrm{b}+\mathrm{th})-\mathrm{TF}(\mathrm{b})-\mathrm{TF}(\mathrm{b}, \mathrm{th})}{\mathrm{t}} \leq \mathrm{TF}(\mathrm{b}+\mathrm{h})-\mathrm{TF}(\mathrm{b})-\mathrm{TF}(\mathrm{b}, \mathrm{h})
$$

and taking now limits when $t \rightarrow 0$, it follows that

$$
\mathrm{TF}(\mathrm{g}(\mathrm{x}))-\mathrm{TF}^{\prime}(\mathrm{b}, \mathrm{g}(\mathrm{x})) \geq \mathrm{TF}(\mathrm{b})-\mathrm{TF}(\mathrm{b}, \mathrm{b}) \text {. }
$$

Now, since $x$ is a feasible solution of $(2, \lg (x))$, we have that $\operatorname{TF}(\mathrm{g}(\mathrm{x})) \leq \operatorname{Tf}(\mathrm{x})$ and therefore

$$
\operatorname{Tf}(\mathrm{x})-\mathrm{TF}^{\prime}(\mathrm{b}, \mathrm{g}(\mathrm{x})) \geq \mathrm{TF}(\mathrm{b})-\mathrm{TF}(\mathrm{b}, \mathrm{b}) \text {. }
$$

From (3.1) and (3.2) it is immediately deduced that $-\mathrm{F}^{\prime}(\mathrm{b},-) \in \Gamma_{\mathrm{T}}$ and
$\varphi\left(\mathrm{T},-\mathrm{TF}^{\prime}(\mathrm{b},-)\right) \geq \mathrm{TF}(\mathrm{b})-\mathrm{TF}^{\prime}(\mathrm{b}, \mathrm{b})$.

To prove the opposite of inequality (3.3) it is enough to see that the inequality of (3.2) changes its sense if $x=x_{b}$, and this is a consequence of the inequality $-T F^{\prime}\left(b, g\left(x_{b}\right)\right) \leq-T F^{\prime}(b, b)$, which follows immediately from $g\left(x_{b}\right) \leq b$ and $-\mathrm{TF}^{\prime}(\mathrm{b},-) \geq 0$.

Suppose now that 3.1.2 holds and let $b \in \mathrm{B}$ be. Then it follows from Theorem 10 of [4] that TG(b) is a Lagrangian T-multiplier of $(2,1 b)$ (in the sense of Definition 1

of [4]) and therefore, since $x_{b}$ is a T-optimal solution of $(2,1 b)$, we have that

$\mathrm{TF}(\mathrm{b})=\operatorname{Tf}\left(\mathrm{x}_{\mathrm{b}}\right)=\operatorname{Inf}\{\operatorname{Tf}(\mathrm{x}) ; \mathrm{x} \in \Omega, \mathrm{g}(\mathrm{x}) \leq \mathrm{b}\}$

$=\operatorname{Inf}\{\operatorname{Tf}(x)+\operatorname{TG}(b)(g(x)-b) ; x \in \Omega\}$

$=\operatorname{Inf}\{\operatorname{Tf}(x)+\operatorname{TG}(b)(g(x)) ; x \in \Omega\}-T G(b)(b)$.

Moreover, it follows from Theorem 4 of [4] that TF' $(b,-)=-T G(b)$, and thus it is obtained from (3.4) that $\mathrm{TF}(\mathrm{b})=\varphi(\mathrm{T},-\mathrm{TF}(\mathrm{b},-))+\mathrm{TF}^{\prime}(\mathrm{b}, \mathrm{b})$.

Definition 3.2. Let $U$ and $V$ be two Banach spaces, $p \in \mathbf{R}$ and $A$ an open subset of $\mathrm{U}$. We say that a function $\mathrm{h}: \mathrm{A} \rightarrow \mathrm{V}$ is homogeneous of degree $\mathrm{p}$ if for every $a \in A$ there exists a neighborhood $U^{a} \subset(0,+\infty)$ of 1 (in the real line $R$ ) such that $\mathrm{ta} \in \mathrm{A}$ and $\mathrm{h}(\mathrm{ta})=\mathrm{t}^{\mathrm{p}} \mathrm{h}(\mathrm{a})$ for every $\mathrm{t} \in \mathrm{U}^{\mathrm{a}}$.

Theorem 3.3. (Euler's Formula). Let $U$ and $V$ be two Banach spaces, $p \in R, A$ an open subset of $U$ and $h: A \rightarrow V$ a Fréchet differentiable function. Then $h$ is homogeneous of degree $p$ if and only if $h^{\prime}(a, a)=p h(a)$ for every $a \in A$

Theorem 3.4. With the already established notations, let us assume that $F$ is Fréchet differentiable on $B$ and that at least one of the conditions 3.1.1 and 3.1.2, holds. Then, $-F^{\prime}(b,-)$ is a dual T-optimal solution associated to $x_{b}$ for every $b \in B$ if and only if $\pi \mathrm{F}$ is homogeneous of degree 1 .

Proof. Suppose that $-F^{\prime}(b,-)$ is associated to $x_{b}$ for every $b \in B$. Then we have that $F(b)=f\left(x_{b}\right)=\psi\left(T,-F^{\prime}(b,-)\right)=T^{*}-1 \varphi\left(T,-T F^{\prime}(b,-)\right)+F^{\prime}(b, b)$ for every $b \in B$, and since clearly $\pi T^{*-1}=0$, applying $\pi$ to the terms of last equalities we obtain that $\pi \mathrm{F}(\mathrm{b})=\pi \mathrm{F}^{\prime}(\mathrm{b}, \mathrm{b})$ and now, it results immediately from the Euler's Formula that $\pi \mathrm{F}$ is homogeneous of degree 1 .

Reciprocally, if $\pi F$ is supposed to be homogeneous of degree 1 then it follows from the Euler's Formula that $\pi \mathrm{F}(\mathrm{b})=\pi \mathrm{F}^{\prime}(\mathrm{b}, \mathrm{b})$. Moreover, it is deduced from Lemma 3.1 that $-F^{\prime}(b,-)$ is a feasible dual solution (and a dual T-optimal solution) and $\varphi\left(T,-T F^{\prime}(b,-)\right)+T F^{\prime}(b, b)=T F(b)$.

Therefore, since $y=T^{*-1} T(y)+\pi(y)$ for every $y \in Y$, it results that

$\psi(\mathrm{T},-\mathrm{TF}(\mathrm{b},-))=\mathrm{T}^{*-1} \varphi\left(\mathrm{T},-\mathrm{F}^{\prime}(\mathrm{b},-)\right)+\mathrm{F}^{\prime}(\mathrm{b}, \mathrm{b})$

$=T^{*}-1\left(T F(b)-T F^{\prime}(b, b)\right)+F^{\prime}(b, b)$

$=F(b)-F^{\prime}(b, b)-\pi F(b)+\pi F^{\prime}(b, b)+F^{\prime}(b, b)=F(b)=f\left(x_{b}\right)$

and thus $-F^{\prime}(b,-)$ is associated to $x_{b}$. 
Remark. Concrete examples at which $-\mathrm{F}^{\prime}(\mathrm{b},-)$ is not a dual solution associated to $x_{b}$, can be fond in [4]. This fact and Theorem 3.4 point out that the homogeneity of $\pi \mathrm{F}$ does not hold in general. In the particular case of the scalar programming clearly $\pi=0$ and so the homogeneity of $\pi \mathrm{F}$ is obvious and this shows that Theorem 3.4 is a genuine extension of the classic results known for the scalar programming.

Definition 3.5. Let $b \in \mathrm{B}$ be and $\mathrm{p}, \mathrm{q} \in \mathbf{R}$. We say that $(2,1 b)$ is a $(p, q)$-homogeneous program if $\Omega$ is a convex cone of $X$ and the equalities $f(t x)=t^{p} f(x)$ and $g(t x)=t^{q} g(x)$ hold for every $x \in \Omega$ and every $0<t(\in \mathbf{R})$. If moreover $p=q$ we merely say that $(2,1 b)$ is $p$-homogeneous.

Proposition 3.6. If the program $(2,1 b)$ is $(p, q)$-homogeneous and $q \neq 0$ then the function TF is homogeneous of degree $\mathrm{p} / \mathrm{q}$.

Proof. It follows easily proceeding in standard way.

Theorem 3.7. Suppose that $F$ is Fréchet differentiable on $B, p \in \mathbf{R}-\{0\}$, the program $(2,1 b)$ is p-homogeneous for every $b \in B$ and at least one of the conditions 3.4.1 and 3.4.2 holds. Then $-F^{\prime}(b,-)$ is a T-optimal solution associated to $x_{b}$ for every $b \in B$ if and only if $F$ is homogeneous of degree 1 .

Proof. It is an immediate consequence of Theorem 3.4 and Proposition 3.6 since $\mathrm{F}=\mathrm{T}^{*}-1 \mathrm{TF}+\pi \mathrm{F}$

Remark. In [3] it is given an example of a linear program for which $-F^{\prime}(b,-)$ is not a dual associated solution and this proves that in general $\mathrm{F}$ is not homogeneous. In the particular case of the scalar programming. Theorem 3.7 states that if the function $F$ is Fréchet differentiable then it is necessarily homogeneous of degree 1 (even for infinite dimensional problems).

\section{Linear programs}

We will say that $(2,1 \mathrm{~b})$ is linear if $\Omega$ is a convex cone of $X$ and the functions $f$ and $g$ can be extended to all $\mathrm{X}$ in such a way that their extensions, which will be also denoted by $\mathrm{f}$ and $\mathrm{g}$, belong to $L(\mathrm{X}, \mathrm{Y})$ and $L(\mathrm{X}, \mathrm{Z})$, respectively. In particular, linear programs are 1-homogeneous and as usual we will write $x \geq 0$ instead of the constraint $\mathrm{x} \in \Omega$.

Throughout this section $(2,1 \mathrm{~b})$ will be supposed to be a linear program for every $b \in B$, and since in linear programing it is more convenient to work with equality constraints, we will change $(2,1 \mathrm{~b})$ by the following program:

$$
\text { Min } f(x)
$$

$\mathrm{g}(\mathrm{x})+\mathrm{z}=\mathrm{b}, \mathrm{x} \in \Omega, \mathrm{z} \in \mathrm{Z}_{+}$

Clearly there is a natural bijection between the feasible sets of $(2,1 \mathrm{~b})$ and $(4,1 \mathrm{~b})$, preserving the optimums, the T-optimal solutions and the extremal points of both feasible sets.

Theorem 14 of $[4]$ proves that if the program $(2,1 b)$ is linear, then its dual has the following more simple formulation:

$$
\operatorname{Max}-\mathrm{G}(\mathrm{b})
$$

$$
\mathrm{G} \in L(\mathrm{Z}, \mathrm{Y}), \mathrm{TG} \geq 0, \mathrm{TGg} \geq-\mathrm{Tf}
$$

Last dual extends the duals given in [1] for scalar linear programs, in [9] for linear multiobjective programs in finite dimensional spaces and in [3] for linear programs between spaces of arbitrary dimensions. Moreover (4.2b) is quite similar to the dual between spaces of arbitrary dimensions. Moreover (4.2b) is quite similar to the dual
introduced in [8] for goal programming problems and also to the dual stated in [10] for general linear problems. Throughout this section we will suppose that for every $b \in B,\left\{x_{b}^{i}\right\}_{i=1,2, \ldots, k}$ is a family of basic feasible solutions (in the sense of [1]) which are T-optimal solutions of $(4,1 b)$. Therefore $\operatorname{Tf}\left(x_{b}^{i}\right)=\operatorname{Tf}\left(x_{b}^{j}\right)$ for every $b \in B$ and $i, j \in\{1, \ldots, k\}$, and every convex linear combination of $\left\{x_{b}^{1}, \ldots, x_{b}^{k}\right\}$ is also a $T$-optimal solution of $(4,2 b)$ for every $b \in B$.

If $\mathrm{z}_{\mathrm{b}}^{\mathrm{i}}=\mathrm{b}-\mathrm{g}\left(\mathrm{x}_{\mathrm{b}}^{\mathrm{i}}\right)$ for every $1 \leq \mathrm{i} \leq \mathrm{k}$ and $\mathrm{b} \in \mathrm{B}$, we have that

$\beta_{0}\left(x_{b}^{i}\right)=\left\{(x, z) \in X x Z:\left(x_{b}^{1}, z_{b}^{1}\right)+\lambda(x, z),\left(x_{b}^{1}, z_{b}^{1}\right)-\lambda(x, z) \in \Omega \times Z_{+}\right.$ for some scalar $\lambda>0\}$ is a vector subspace of $X x Z$ such that $\left(x^{i}, z_{b}^{i}\right) \in \beta_{0}\left(x_{b}^{i}\right)$ and $\beta_{0}\left(x_{b}^{1}\right) \cap$ Ker $g^{*}=\{0\}$, where $g^{*}: X x Z \rightarrow Z$ is defined by $g^{*}(x, z)=g(x)+z$ for every $(x, z) \in X x Z$. We will assume that $\beta_{0}\left(x_{b}{ }_{b}\right)$ is contained in a closed subspace $\beta_{i}$ which is a topological supplement of $\operatorname{Ker} g^{*}$ and it does not depend on $b \in B$

Clearly, if we assume in this section that $\mathrm{g}$ is surjective then the restriction $\mathrm{g}_{\mathrm{i}}{ }^{*}$ of $\mathrm{g}^{*}$ to $\beta_{i}$ is a topological isomorphism such that $g_{i}^{*}\left(x_{b}^{i}, z_{b}^{i}\right)=b$ for every $i=1, \ldots, k$ and $\mathrm{b} \in \mathrm{B}$

Consider also $k$ functions $h_{i}: B \rightarrow[0,1](i=1, \ldots, k)$ such that $\sum_{i=1}^{k} h_{i}(b)=1$ for every $b \in B$, and let us fix the following notations: $x_{b}=\sum_{i=1}^{k} h_{i}(b) x_{b}^{i}$, $F(b)=f\left(x_{b}\right)$ and $z_{b}=b-g\left(x_{b}\right)=\sum_{i=1}^{k} h_{j}(b)\left(b-g\left(x_{b}^{i}\right)\right)=\sum_{i=1}^{k} h_{i}(b) z_{b}^{i}$ for every $b \in B$. Finally assume throughout this section that at least one of the conditions 3.1 .1 and 3.1 .2 , holds.

Proposition 4.1. If $F$ is Fréchet differentiable at $B$ and $h_{i}(i=1, \ldots, k)$ is homogeneous of degree 0 , then $-F^{\prime}(b,-)$ is a dual T-optimal solution associated to $x_{b}$ for every $b \in B$.

Proof. It follows from Theorem 3.7 that it is enough to prove that the function $F$ is homogeneous of degree 1 , and this can be easily proved in standard way.

Theorem 4.2. Suppose that the functions $F$ and $h_{i}(i=1, \ldots, k)$ are Fréchet differentiable on $B$ and $\left\{f\left(x_{b}^{i}\right) ; i=1, \ldots, k\right\}$ is a linearly independent system for every $b \in B$. Then $-F^{\prime}(b,-)$ is a dual $T$-optimal solution associated to $x_{b}$ for every $b \in B$ if and only if $h_{i}$ is homogeneous of degree 0 for every $i=1, \ldots, k$. 
Proof. Like in the proof of Proposition 4.1, let us denote by $f$ the natural extension of $f$ to $X x Z$ (such that $(x, z) \rightarrow f(x))$. If $-F^{\prime}(b,-)$ is a dual T-optimal solution associated to $x_{b}$ for every $b \in B$, then it results from Theorem 3.7 that $F$ is homogeneous of degree 1 and then it follows from the Euler's Formula that $F(b)=F^{\prime}(b, b)$ for every $b \in B$. Furthermore

$$
F(b)=f\left(x_{b}, z_{b}\right)=f\left(\sum_{i=1}^{k} h_{i}(b)\left(x_{b}^{i}, z_{b}^{i}\right)\right)=f\left(\sum_{i=1}^{k} h_{i}(b) g_{i}^{*-1}(b)\right)
$$

and

$$
\begin{aligned}
& F^{\prime}(b, b)=f\left(\sum_{i=1}^{k} h_{i}(b, b) g_{i}^{*-1}(b)+\sum_{i=1}^{k} h_{i}(b) g_{i}^{*-1}(b)\right) \\
& =f\left(\sum_{i=1}^{k} h_{i}(b, b) g_{i}^{*-1}(b)\right)+f\left(\sum_{i=1}^{k} h_{i}(b) g_{i}^{*-1}(b)\right)
\end{aligned}
$$

for every $b \in B$.Therefore since $F(b)=F^{\prime}(b, b)$ for every $b \in B$, it results from (4.1) and (4.2) that

$$
0=\sum_{i=1}^{k} h_{i}^{\prime}(b, b) f\left(g_{i}^{*-1}(b)\right)=\sum_{i=1}^{k} h_{i}^{\prime}(b, b) f\left(x_{b}^{i}, z_{b}^{i}\right)
$$

for every $b \in B$, from where it is immediately deduced that $h_{i}^{\prime}(b, b)=0$ for every $b \in B$ and $i=1, \ldots, k$, since the system $\left\{f\left(x_{b}^{i}, z_{b}^{i}\right) ; i=1, \ldots, k\right\}$ is linearly independent. Now it results from the Euler's Formula that $h_{i}$ is homogeneous of degree 0 for every $i=1, \ldots, k$. The converse follows trivially from Proposition 4.1.

Remark. The assumption about the linearly independence of the system $\left\{f\left(x_{b}^{i}, z_{b}^{i}\right) ; i=1, \ldots, k\right\} \quad(b \in B)$ cannot be avoid in Theorem 4.2 since it is known that in the scalar programming (i.e., $Y=R$ ) the differential $-F^{\prime}(b,-)$ is the dual solution independently of the choice of the primal optimum. Actually what happens in this case is that $F$ does not depend on the "weights" $h_{j}$ applied to every basic feasible solution.

Many papers (like [3], [6] and [9] ) prove that if the decisor chooses an optimum solution which is a basic feasible solution (or a convex linear combination of basic feasible solutions whose scalar coefficients are fixed and independent of $b \in B$ ) then $-F^{\prime}(b,-)$ can be fond as a dual associated solution. Theorem 4.2 states that $-F^{\prime}(b,-)$ continues being a dual solution associated to $x_{b}$ even though the weights are not constant and they are homogeneous functions ( $\mathrm{f} b$ ) of degree 0

\section{The basic differential equation}

Let us remark that if $-F^{\prime}(b,-)$ is a T-optimal solution associated to $x_{b}$, for every $\mathrm{b} \in \mathrm{B}$, and we consider the function $\mathrm{G}: \mathrm{B} \rightarrow L(\mathrm{Z}, \mathrm{Y})$ such that $\mathbf{G}(b)=-F^{\prime}(b,-)$ for every $b \in B$, then it follows from Theorem 12 of [4] that if $\mathrm{G}$ is Fréchet differentiable (or equivalently, if $\mathrm{F}$ is twice Fréchet differentiable) then

$$
(\pi \mathrm{G}) \cdot(b, z)(b)=0
$$

for every $b \in B$ and $z \in Z$. Thus in many situations (stated in Sections 3 and 4 ) the differential equation (5.1) (jointly with some initial and optimal conditions) let to obtain $F^{\prime}$. In particular, if $Y=\mathbf{R}^{\mathrm{m}}$ and $Z=\mathbf{R}^{\mathrm{n}}$, then $\pi \mathbf{G}$ (b) will be a functional matrix $\left(\mathrm{g}_{\mathrm{ij}}(\mathrm{b})\right)_{\mathrm{i}=1, \ldots, \mathrm{m}, \mathrm{j}=1, \ldots, \mathrm{n}}$, and (5.1) leads to the following system of partial differential equations:

$$
\sum_{j=1}^{n} b_{j} \frac{\partial g_{i j}}{\partial b_{k}}=0 \quad i=1, \ldots, m, k=1, \ldots, n .
$$

\section{Conclusions}

If $\mathrm{b}$ denotes the right side vector of the constraints of a convex multiobjective program and $F(b)$ is the optimum value of that program, then the differential of $F$ with respect to $b$ cannot be obtained in general as a solution of a dual program (see [3] or [4]) and it appears the natural question of characterizing the situations where this differential (denoted by $F^{\prime}(b,-)$ in this paper) solves the dual program. Theorem 3.4 gives an answer to this question by means of a condition of homogeneity, and Theorem 3.7 states for a particular type of programs (containing the linear ones) that a necessary and sufficient condition to be $-F^{\prime}(b,-)$ an associated dual solution is that $\mathrm{F}$ will be (as a function of $\mathrm{b}$ ) homogeneous of degree 1 . In particular, for the scalar linear programming it is verified that if $F$ is differentiable then it is necessarily homogeneous of degree 1 , result which is false in general in the multiobjective case.

In the linear case, when the optimum solution is chosen to be a convex linear In the linear case, when the described between finite dimensional spaces, and also in many examples of infinite dimensional programs like the Mass-Transfer type problems) the necessary and sufficient condition for $-F^{\prime}(b,-)$ to be an associated dual solution is that the weights applied on the basic feasible solutions will be homogeneous functions (of b) of degree 0 (as Theorem 4.2 states)

Last results can be proved, for programs described between finite dimensional spaces, solving the Euler's partial differential equations which characterize the homogeneous functions. Furthermore, if $F$ is homogeneus of degree 1 , then the system of partial differential equations (5.2) may lead in practical situations to a general expresion of $\mathrm{F}$. When $\mathrm{F}$ is not a homogeneous function (of $b$ ) then the sensitivity of the program can be obtained solving a more general and complex system of partial differential equations which can be deduced from Theorem 12 of [4]

\section{References}

[1] Anderson, E.J. and P. Nash: Linear programming in infinite dimensional spaces. John Wiley \& Sons, New York, 1987.

[2] Balbás, A., F. Fernández, and P. Jiménez Guerra: On the envolvent theorem in multiobjective programming. Indian Journal of Pure and Applied Mathematics, 26 (1995), 1035-1047

3]Balbás, A. and A. Heras,: Duality theory for infinite dimensional multiobjective programming. European Journal of Operational Research, 68 (1993), 379-388. 
[4] Baibás, A. and P. Jiménez Guerra: Sensitivity analysis for convex multiobjective programming in abstract spaces. Journal of Mathematical Analysis and Applications, 202 (1996), 645-658

[5] Balbás, A., P. Jiménez Guerra and C. Núñez: Strongly proper optimums and maximal optimization in multiobjective programming. Revista Real Academia de Ciencias de Madrid, 86 (1992), 289-295.

[6] Henggeler, C. and J. Cimaco: Sensitivity analysis in MCDM using the weight space. Operations Research Letters, 12 (1992), 187-196.

[7] Ignizio, J.P.: Introduction to linear goal programming. Sage University Papers, London, 1976.

[8] Ignizio, J.P. : An algorithm for solving the linear goal programming problem by solving its dual. Journal of the Operations Research Society, 36 (1985), 507-515.

[9] Isermann, H.: On some relations between a dual pair of multiobjective linear programs. Zeitschrift für Operations Research, 22 (1978), 33-41.

[10] Jahn, J.: Mathematical vector optimization in partially ordered linear spaces. Verlag Peter Lang, Frankfurt, 1986.

[11] Jiménez Guerra, P. and B. Rodríguez-Salinas: A general solution of the Monge-Kantorovich Mass-Transfer problem. Journal of Mathematical Analysis and Applications, 202 (1996), 402-510.

[12] Khanh, P.O.: Sufficient optimality conditions and duality in vector optimization with invex-convexlike functions. Journal of Optimization. Theory and Applications, 87 (1995), 359-368.

[13] Khanh, P.O.: Proper solutions of vector optimization problems. Journal of Optimization. Theory and Applications, 74 (1993), 105-130.

[14] Klose, J.: Sensitivity analysis using the tangent derivative. Numerical Functional Analysis and Optimization, 13 (1992), 143-153.

[15] Luc, D.T.: About duality and alternative in multiobjective optimization. Journal of Otimization. Theory and Applications, 53 (1987), 303-307.

[16] Schaefer, H.H.: Banach lattices and positive operators. Springer-Verlag, Berlin, 1974.

[17] Tanino, J., Y. Sawaragy and Y. Nakayama: Theory of multiobjective optimization. Academic Press, 1985.

[18] Zeleny, Y.: Multiobjective linear programming. Springer-Verlag, Berlin, 1974

[19] Zowe, S.: A duality theorem for a convex programming problem in orde complete vector lattices. Journal of Mathematical Analysis and Applications, 50 (1975), 273-287 